\title{
Gravitational Capture of Cosmic Strings by a Black Hole *
}

\author{
Jean-Pierre De Villiers $^{\dagger 1}$, and Valeri Frolov ${ }^{\ddagger 1,2,3}$
}

October 17, 2018

1 Theoretical Physics Institute, Department of Physics, University of Alberta, Edmonton, Canada T6G 2J1

${ }^{2}$ CIAR Cosmology Program

${ }^{3}$ P.N.Lebedev Physics Institute, Leninskii Prospect 53, Moscow 117924, Russia

\begin{abstract}
The gravitational interaction of an infinitely long cosmic string with a Schwarzschild black hole is studied. We consider a straight string that is initially at a great distance and moving at some initial velocity $v(0<v<c)$ towards the black hole. The equations of motion of the string are solved numerically to obtain the dependence of the capture impact parameter on the initial velocity.
\end{abstract}

PACS number(s): 04.60.+n, 12.25.+e, 97.60.Lf, 07.05.Tp

\footnotetext{
*Preprint Alberta Thy 14-97

†e-mail: jpd@phys.ualberta.ca

†e-mail: frolov@phys.ualberta.ca
} 


\section{Introduction}

Cosmic strings are topologically stable one-dimensional objects which are predicted by grand unified theories. Cosmic strings (as well as other topological defects) may appear during a phase transition in the early Universe. (A detailed discussion of cosmic strings and other topological defects can be found in Shellard and Vilenkin [1].) The characteristic thickness of a cosmic string is $\rho_{0} \sim l_{\mathrm{Pl}}\left(m_{\mathrm{GUT}} / m_{\mathrm{Pl}}\right)$ while its mass per unit length is $\mu \sim\left(m_{\mathrm{Pl}} / l_{\mathrm{Pl}}\right)\left(m_{\mathrm{GUT}} / m_{\mathrm{Pl}}\right)^{2}$. Here $m_{\mathrm{GUT}}$ is the characteristic mass defining the symmetry breaking in the grand unified theory which is responsible for the cosmic string formation, and $m_{\mathrm{Pl}}$ and $l_{\mathrm{Pl}}$ are the Planck mass and length, respectively.

For cosmic strings of astrophysical interest the parameter $\rho_{0}$ is much less than any other parameters that enter the problem, such as the length of the string or the size of inhomogeneity of the gravitational field in which it is moving, so that one can idealize the motion of the string as a two-dimensional worldsheet $x^{\mu}=x^{\mu}(\tau, \sigma)$. It can be shown that the equations of motion of a cosmic string in this approximation follow from the Nambu-Goto action (see e.g., Shellard and Vilenkin [1]),

$$
I=-\mu \int d^{2} \xi \sqrt{-\operatorname{det}(G)}, \quad G_{A B}=g_{\mu \nu} \frac{\partial x^{\mu}}{\partial \xi^{A}} \frac{\partial x^{\nu}}{\partial \xi^{B}}
$$

where $g_{\mu \nu}$ is the spacetime metric, $\xi^{A}$ denote the worldsheet coordinates $\left(A, B=0,1 ; \xi^{0}=\tau, \xi^{1}=\sigma\right)$, and $G_{A B}$ is the induced metric on the worldsheet of the string. A two-dimensional worldsheet which provides an 
extremum to the Nambu-Goto action is a minimal surface.

We consider the interaction of a cosmic string with a black hole of mass $M$. For this problem the gravitational field created by the string can be neglected because the dimensionless parameter which characterizes the strength of the field $\mu^{*}=G \mu / c^{2}$ is negligibly small (for GUT one has $\mu^{*} \sim 10^{-6}$ ). It means that we can consider the string as a test object moving in the background of a black hole metric. We also assume that the length of the string $L$ is much greater than the gravitational radius, $L \gg r_{\mathrm{g}}=2 G M / c^{2}$, and use the infinite-string approximation.

There are two possible outcomes for a cosmic string moving from infinity towards a black hole: it may pass near the black hole and escape to the distant region (gravitational scattering), or it may be captured by the black hole. The scattering of a string is an inelastic process since the string absorbs some energy (i.e. the string's internal energy, connected with the excitations of its degrees of freedom, changes during the interaction). The capture of a cosmic string occurs when the string passes sufficiently close to the gravitational radius. After capture, the string remains attached to the black hole. For $\mu L \ll M$ the total mass of the string is much smaller than the mass of the black hole, and the attached string will move around the black hole while the black hole remains practically at rest. In the opposite case, $\mu L \gg M$, the black hole will be accelerated by the string. The characteristic time of this process is $T \sim v M /\left(\mu c^{2}\right) \sim(v / c)\left(\mu^{*} r_{\mathrm{g}} / c\right)$.

Our aim is to study the gravitational interaction of a cosmic string with 
a Schwarzschild black hole and to determine the conditions under which the string is captured. For simplicity, we consider an infinitely long string which is initially straight (far from the black hole), and denote its initial velocity by $v$ and its impact parameter by $b$ (see Figure 1). For a given velocity $v$, capture occurs if $b$ is less than the so-called capture impact parameter $b_{\text {capture }}(v)$. By solving the equations of motion of the string numerically we obtain the dependence of the capture impact parameter on the initial velocity $v$.

\section{Equations of Motion}

In order to use numerical methods it is convenient to deal with a string of finite size. For this purpose we modify the problem by taking a finite string and attaching heavy particles ("monopoles") at its ends (see Figure 1). In this case, it is easier to use a quadratic form of the string action, equivalent to the Nambu-Goto action, namely the Polyakov action [2]. The corresponding action takes the form,

$$
\begin{aligned}
S\left[x^{\mu}, h_{A B}, \sigma_{i}\right] & =-\mu \int_{\tau_{1}}^{\tau_{2}} d \tau \int_{\sigma_{1}(\tau)}^{\sigma_{2}(\tau)} d \sigma \sqrt{-h} h^{A B} G_{A B} \\
& -\sum_{i=1}^{2} \frac{m_{i}}{2} \int_{\tau_{1}}^{\tau_{2}} d \tau g_{\mu \nu} \frac{d X_{i}^{\mu}(\tau)}{d \tau} \frac{d X_{i}^{\nu}(\tau)}{d \tau}
\end{aligned}
$$

where $h_{A B}$ is the internal metric with determinant $h$, and $X_{i}^{\mu}(\tau)=x^{\mu}\left(\tau, \sigma_{i}(\tau)\right)$ is a world-line of the $i$-particle, and $m_{i}$ is its mass. The end points are taken to represent the mass of a longer (potentially infinite) string lying outside the region of interest; the motion of these points represents a boundary condition 
for the section of the string worldsheet under study. This approach allows us to approximate the motion of an infinite string by considering the case of extremely heavy particles, when $m_{i} \rightarrow \infty$. Under this assumption, the variation of the action of the massive end points with respect to $X_{i}^{\mu}(\tau)$ leads to the equations of motion,

$$
\frac{d^{2} X_{i}^{\mu}(\tau)}{d \tau^{2}}+\Gamma_{\alpha \beta}^{\mu} \frac{d X_{i}^{\alpha}(\tau)}{d \tau} \frac{d X_{i}^{\beta}(\tau)}{d \tau}=0 .
$$

The equations of motion of a string are obtained by varying the action (2) with respect to $x^{\mu}(\tau, \sigma)$. We fix the freedom in the choice of internal coordinates $(\tau, \sigma)$ on the worldsheet of the string by using a conformal gauge so that $h_{A B}$ is of the form $h_{A B}=h \operatorname{diag}(-1,1)$. The remaining freedom is used to put $\sigma_{1}=-\pi / 2$ and $\sigma_{2}=\pi / 2$ for the end points. In these coordinates the equations of motion of the string have the form

$$
\begin{aligned}
\frac{\partial^{2} x^{\mu}}{\partial \tau^{2}}-\frac{\partial^{2} x^{\mu}}{\partial \sigma^{2}}+\Gamma_{\rho \eta}^{\mu} \frac{\partial x^{\rho}}{\partial \tau} \frac{\partial x^{\eta}}{\partial \tau}-\Gamma_{\rho \eta}^{\mu} \frac{\partial x^{\rho}}{\partial \sigma} \frac{\partial x^{\eta}}{\partial \sigma} & =0\left(-\frac{\pi}{2}<\sigma<\frac{\pi}{2}\right) \\
g_{\mu \nu}\left(\frac{\partial x^{\mu}}{\partial \tau} \frac{\partial x^{\nu}}{\partial \tau}+\frac{\partial x^{\mu}}{\partial \sigma} \frac{\partial x^{\nu}}{\partial \sigma}\right) & =0 \\
g_{\mu \nu} \frac{\partial x^{\mu}}{\partial \tau} \frac{\partial x^{\nu}}{\partial \sigma} & =0
\end{aligned}
$$

The last two equations are constraints. We use them as checks on the quality of the numerical solutions to (4).

The equations of motion of the string and the end points need to be solved simultaneously. In order to do this, it is necessary to relate the proper time of the string $(\tau)$ and the parameter $(\tau)$ appearing in (3). This is done in the process of performing variation of the action for the finite length of string, 
which yields conditions on the boundaries of the string worldsheet. The details of these calculations are not important, but it follows from matching the behaviour of the boundaries of the string worldsheet and the massive particles that these two time-like parameters must be the same, namely the proper time. So, together with the equations of motion for the string (4), the condition that the end points of the string move along geodesics,

$$
x^{\mu}\left(\tau,-\frac{\pi}{2}\right)=X_{1}^{\mu}(\tau), \quad x^{\mu}\left(\tau, \frac{\pi}{2}\right)=X_{2}^{\mu}(\tau),
$$

gives the boundary conditions for the string equations.

We assume that the ends of the string are moving on initially parallel geodesics with velocity $v$. Numerical results demonstrate that as soon as the length $L$ of the string becomes greater than some quantity $L_{m}(v)$ the capture

parameter $b_{\text {capture }}(v)$ does not depend on $L$. In other words, in this regime the massive end points do not alter the dynamics of the string itself. Their role becomes one of simulating the behaviour of an infinite string. More will be said on this in the following sections.

\section{$3 \quad$ Numerical Method}

The equations of motion of the cosmic string have the form of a system of non-linear wave equations. After some trials with more traditional approaches, a method based on Von Neumann's discretization for the linear wave equation was developed. This approach deals with the wave equation directly as a second-order equation, with a standard finite differencing for the time derivative and an averaging method for the spatial derivative that gives 
rise to an implicit scheme. Von Neumann's discretization yields a tridiagonal system that is solved algebraically. The extended version of the method deals with a block-tridiagonal system of linearized equations that is solved iteratively. Details on the numerical method can be found in [3].

In order to have a well-posed problem, we must specify the shape of the string at some initial time $\tau_{0}$, along with normal derivatives $\left(\partial_{\tau} x^{\mu}\right)$ everywhere on the string, including the massive ends, at this initial time. Taken together, these completely specify the initial/boundary value problem (IBVP). Since the Von Neumann method requires two time steps in order to compute the next (unknown) time step, a completely equivalent way of specifying the IBVP is to use two initial time steps. Using the property of asymptotic flatness for the Schwarzschild spacetime, and the fact that analytic solutions to the equations of motion in Minkowski spacetime are readily obtained, the IBVP can be specified using analytic expressions. Although this approach requires that the solution be started well away from the black hole, it greatly simplifies the process of starting the Von Neumann solver. Although a cosmic string cannot be straight when exposed to an inverse-square force, the choice of the initial position of the string is made so as to minimize the discrepancy between the analytic (straight) solution and "true" solution in the black hole spacetime; the constraint equations are a useful guide in this choice. The initial position must also be sufficiently distant so that the capture impact parameter is not affected (starting a straight string solution too close to the black hole could have a marked effect on the capture impact parameter). 


\section{The Capture of Cosmic Strings}

The portion of the cosmic string that passes closest to the black hole will experience a larger gravitational force than more distant parts of the string. Because of this, the string will become deformed as it moves through the spacetime of the black hole. For more distant parts of the string, where the gravitational field is weak, it is possible to describe this deformation by solving the linearized Nambu-Goto equations. In this approximation the deformation can be treated as a perturbation to a straight string solution and decomposed into two components: (1) a component that is directed along the original direction of motion, and (2) a component that is orthogonal to (1) and to the straight string itself. This analysis shows that, due to the long-range nature of the gravitational field, the deformation in the direction of motion (1) can be large. What is important is that the deformation along (2) remains finite; this deformation is related to the process of string capture. The deformation of the central part of the sting determines whether or not capture will occur, and a numerical solution to the equations of motion is required to make this determination.

Because the string has tension, which acts to straighten its bent parts, it is reasonable to expect that it would be more difficult to capture the string than a test particle moving with the same velocity and impact parameter. In other words, the capture impact parameter for a string approaching a black hole should be less than the capture impact parameter for a test particle. 
The results of the numerical calculations are presented in Figure 2. It shows $\log -\log$ graphs of the capture impact parameter $\left(b(v) / r_{g}\right)$ as a function of initial velocity. Dashed, dotted, and dashed-dotted lines represent capture impact parameters for strings of lengths $L=100 r_{g}, 1000 r_{g}$, and $2000 r_{g}$, respectively. The capture curves for the cosmic strings are shown with error bars. The lower bound indicates largest impact parameter resulting in capture, while the upper bound indicates the smallest impact parameter for which the string escaped. The data for these curves was obtained using Eddington-Finkelstein ingoing coordinates since it is easy to determine when the string becomes trapped (capture occurs when the radial coordinate in the equatorial plane is less than the gravitational radius).

The graphs show that for a string of finite size the capture impact parameter depends on both the length and velocity of the string, $b(v, L)$. The capture curve for the $L=2000 r_{g}$ string is definitive for velocities $v \geq 0.2 c$, meaning that the capture curves for longer strings are indistinguishable in this velocity range. Probing the low-velocity behaviour of very long strings is numerically intensive, so the extension of the capture curves below $v \approx 0.05 c$ has yet to be carried out.

The solid line represents the capture impact parameter for a test particle. The gravitational capture of test particles is well understood: a par-

\footnotetext{
${ }^{1}$ The gravitational capture of strings by Schwarzschild black holes has already been discussed by Moss and Lonsdale [5]. The results shown above do not agree with these earlier results. The smallest capture impact parameter for a string quoted by these authors occurs at the ultra-relativistic limit, and their capture graph does not reproduce features shown in Figure 2.
} 
ticle moving at non-relativistic velocities has a capture impact parameter $b(v) / r_{g}=2 / v$, while in the ultra-relativistic regime $(v \rightarrow c)$, the capture impact parameter is $b(v) / r_{g}=3 \sqrt{3} / 2$. The capture impact parameter for the ultra-relativistic particle is also the smallest capture impact parameter.

The capture curve of a cosmic string is different in many ways from that of a test particle. Unlike a test particle, the smallest capture impact parameter for a string occurs at some intermediate velocity (e.g $v \approx 0.1 c$ for the $L=$ $2000 r_{g}$ string). The capture impact parameter increases as $v \rightarrow c$ and $v \rightarrow 0$. Also, the smallest capture impact parameter decreases with increasing string length. At a sufficiently low velocity, the capture curve of a (finite) string intersects the curve for the test particle. The capture impact parameter for strings is independent of string length for velocities approaching $c$, even for very short strings.

The increase in the capture impact parameter for velocities approaching the speed of light can be understood in terms of the propagation time of influences along the string. As $v \rightarrow c$, the interaction time where the string experiences the gravitational pull of the black hole becomes progressively shorter, and the influence of the black hole is felt by a shorter and shorter segment of string. It is reasonable to expect that in the ultimate limit, the string will behave like a particle and hence have the same capture impact parameter.

This result illustrates how the internal tension in the string helps determine its response to the gravitational field of the black hole. In the extreme 
relativistic limit, tension plays no role since signals cannot propagate sufficiently far down the string to help influence the capture process. At lower velocities, tension plays an increasingly dominant role and helps the string avoid capture. The smallest capture impact parameter for the string is far less than that for a test particle, and occurs away from the ultra-relativistic limit.

The capture curve for $L=100 r_{g}$ intersects the capture curve for the test particle. This reflects the fact that for small velocities the finiteness of the string plays an important role. The trajectories of the massive particles terminating the strings are focused by the black hole. When the strings are sufficiently long, this focusing is negligible. However, the boundary conditions used in the numerical work force these massive particles to move on geodesics, so they are subject to the same capture characteristics as free test particles. This means that the end particles will be trapped by the black hole when the initial velocity becomes sufficiently small, no matter how far they are initially from the black hole. Since there is a string connecting the two particles, its behaviour will be greatly influenced by a strong focusing of the end points, and hence easily captured. For this reason, the intersection of particle and string curves is to be expected for any finite length of string. However, the initial velocity for which intersection occurs decreases with increasing string length. In the ultimate limit, where string length becomes infinite, the two curves should intersect only when $v=0$.

When $v \rightarrow 0$, the string is expected to exhibit the characteristics of a 
stationary string, provided that the characteristic time for propagation of signals along the string, $t_{\text {prop }} \sim L / c$, is much less than the characteristic time for passage by the black hole $t_{\text {pass }} \sim r_{g} / v$, or by rearranging, the string is effectively static provided that $v / c \ll r_{g} / L$. Stationary string solutions have been discussed by Frolov et al [四]. Using their results, it is easy to show that the minimum radial coordinate of a stationary string of length $\mathrm{L}$ whose end points are located at $\left(X_{0}, Y_{0}, \pm L / 2\right)$ is given by,

$$
r_{\text {min }} \approx r_{g}+\left(\frac{L^{2}+4 Y_{0}^{2}}{8 r_{g}}\right)\left[\frac{\pi}{2}-\arctan \frac{L}{2 Y_{0}}\right]
$$

Provided $r_{\text {min }}>r_{g}$ the string avoids capture. However, it is easily seen that $r_{\min } \rightarrow r_{g}$ as $L \rightarrow \infty$, so that stationary strings of infinite length are always captured. This confirms the claim made above.

\section{Conclusion}

In this numerical study of the gravitational capture of cosmic strings, we modelled strings of infinite length using finite strings with special (geodesic) boundary conditions. These boundary conditions proved problematic at low velocities if the string length is made too short, since the trajectory of the massive end points can be greatly influenced by the black hole under these conditions (the focusing problem). However, the capture curve is independent of string length for velocities close to that of light. Further, a string of infinite length is always harder to capture than a test particle except at the two extremes, $v \rightarrow 0$ and $v \rightarrow c$, where the two capture curves converge. 
Work is currently underway to obtain the capture curves for strings and Kerr (rotating) black holes.

Acknowledgements: This work was partly supported by the Natural Sciences and Engineering Research Council of Canada. One of the authors (V.F.) is grateful to the Killam Trust for its financial support. The authors wish to thank Dr. J.C. Samson, Chair, Dept. of Physics, University of Alberta, for granting access to a Silicon Graphics Power Challenge parallel computer on which all numerical work was carried out.

\section{References}

[1] E. P. S. Shellard and A. Vilenkin, Cosmic Strings. (Cambridge Univ. Press, Cambridge) (1994).

[2] A.M. Polyakov, Phys. Lett., B103, 207-210 (1981).

[3] De Villiers, J.P., An extension of Von Neumann's discretization to a system of non-linear wave equations. Preprint, Alberta Thy 13-97.

[4] Frolov, V.P., Skarzhinsky, V.D., and Zelnikov, A.I. Phys. Lett. B224 255 (1989).

[5] S. Lonsdale and I. Moss, Nucl. Phys. B298, 693 (1988). 


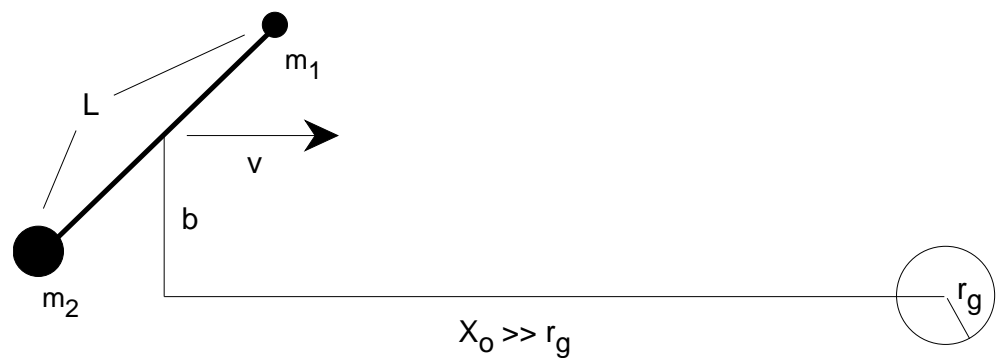

Figure 1: A straight cosmic string moving in the gravitational field of a Schwarzschild black hole. Illustration of the scattering problem. 


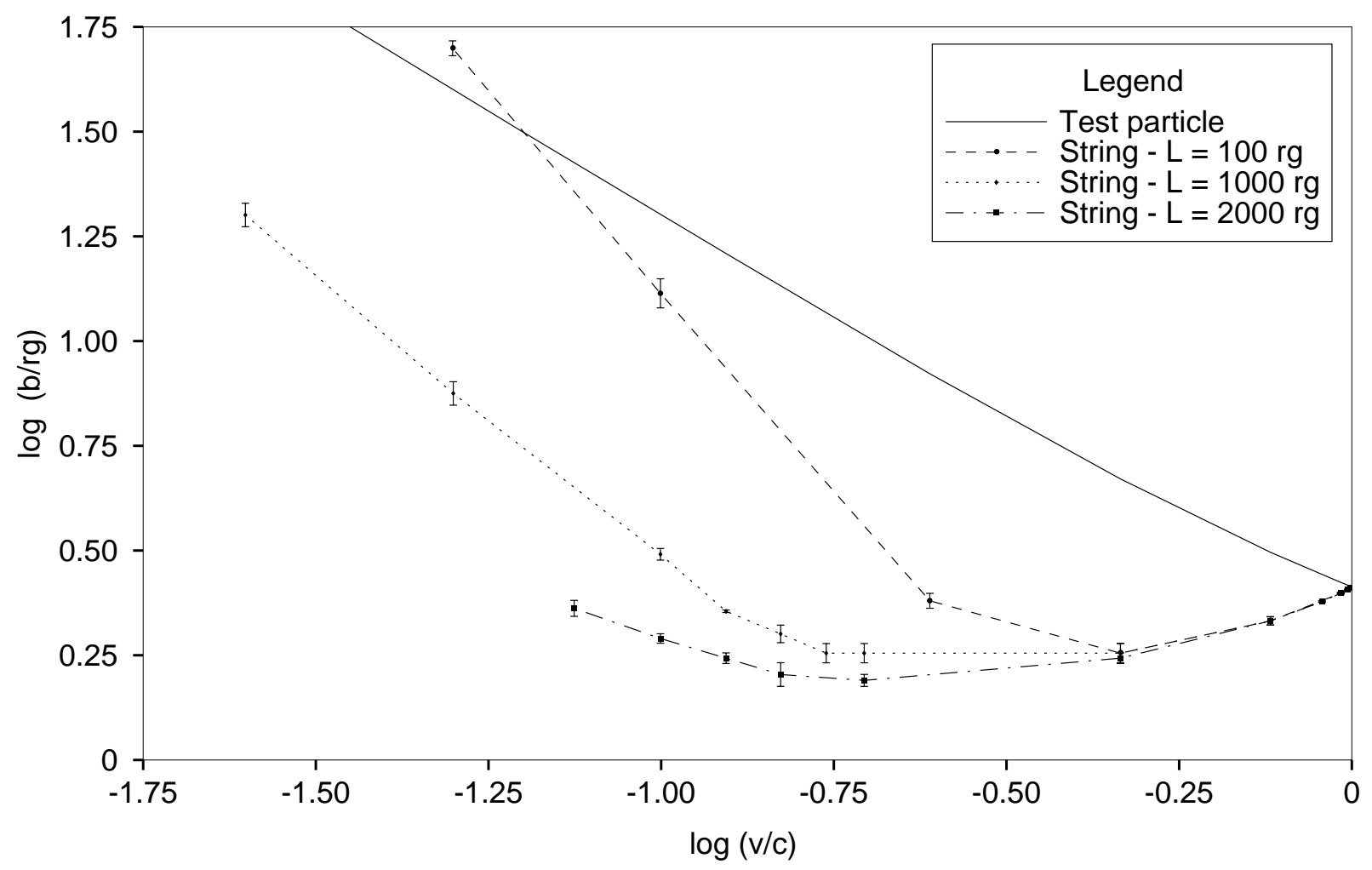

Figure 2: The capture impact parameter $b$ as the function of the initial velocity $v$ of the cosmic string. 